\title{
Use of Latissimus Dorsi Pedicled Myocutaneous Flap for Reconstruction in the Chest Area of an 8-Month-Old Female Infant with Ectopia Cordis
}

\author{
Khaled Dastagir ${ }^{1}$ Thomas Breymann ${ }^{2}$ Andreas Heckmann ${ }^{1} \quad$ Alexander Horke ${ }^{2}$ Peter Maria Vogt ${ }^{1}$ \\ ${ }^{1}$ Department of Plastic, Hand and Reconstructive Surgery, Hannover \\ Medical School, Hannover, Niedersachsen, Germany \\ 2 Department of Pediatric Cardiac Surgery, Hannover Medical School, \\ Hannover, Niedersachsen, Germany \\ Address for correspondence Peter M. Vogt, MD, Dept of Plastic, Hand \\ and Reconstructive Surgery, Hannover Medical School, \\ Carl-Neubergstr. 1, 30625 Hannover, Germany \\ (e-mail: Vogt.Peter@mh-hannover.de).
}

Thorac Cardiovasc Surg Rep 2014;3:38-41.
Abstract
Keywords
- ectopia cordis
- latissimus dorsi pedicled myocutaneous flap
- chest wall reconstruction

Ectopia cordis (EC) is characterized by a complete or partial malposition of the heart outside the thorax. Despite the interdisciplinary treatment, the repair of EC is still very difficult and offers new surgical challenges because of its complexity and various combinations with other anomalies. We report the successful outcome after using a pedicled latissimus dorsi flap in reconstructive surgery in the setting of chronic wound dehiscence in an 8-month-old female infant born with a thoracic EC and omphalocele.

\section{Introduction}

Ectopia cordis (EC) is a rare congenital malposition of the heart outside the thoracic cavity often associated with other serious abnormalities. Because of its complexity and combination with other anomalies, functional and aesthetic repair with reduction of the heart into the thorax cavity, it has not been standardized so far. We present the successful treatment by repair of the complex defect in the anterior chest area in an 8-month-old female infant by repositioning of the heart into the thorax and autologous flap coverage using a pedicled myocutaneous latissimus dorsi flap.

\section{Case Report}

A newborn female infant presented with thoracic EC associated with Cantrell pentalogy, in that the following characteristics were observed: a left ventricular diverticula, a large ventricular septal defect (VSD), atrial septal defect II (ASD II), mitral regurgitation, aortic regurgitation, compensated renal insufficiency, a supraumbilical omphalocele, compensated cardiac insufficiency, cleft of the lower sternum, and complete absence of the xiphoid. Six days after the baby was born, thoracic surgeons repositioned the heart into the thorax cavity. The heart defects, which could be managed medically after tissue coverage, remained unrepaired. Pulmonary artery banding was used to palliate excessive pulmonary flow and failure to thrive; this can usually be deferred when the physiologically elevated pulmonary vascular resistance of the newborn falls to normal values. The thorax wall defect was covered with a Gore-Tex (W. L. Gore \& Associates, Flagstaff, Arizona, United States) mesh and local skin.

However, the skin broke down repeatedly and finally the lack of local tissue and exposure of the mesh jeopardized the proper reposition of the heart. A wound dehiscence was evident at the anatomic level of a xiphoid in the chest area (-Fig. 1). The heart had been previously relocated into the thoracic cavity and reconstruction of the chest wall was achieved using a Gore-Tex Patch (W. L. Gore \& Associates).

Three subsequent attempts to close the wound by secondary sutures failed. Even further dehiscence occurred due to the tension on the wound caused by breathing, the underlying heart beating, the distally located omphalocele, and other muscular contractions especially when the baby cried. received

January 5, 2014

accepted

February 3, 2014

published online

May 29, 2014
DOI http://dx.doi.org/

10.1055/s-0034-1373669. ISSN 2194-7635.

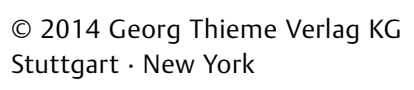

License terms

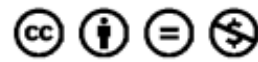




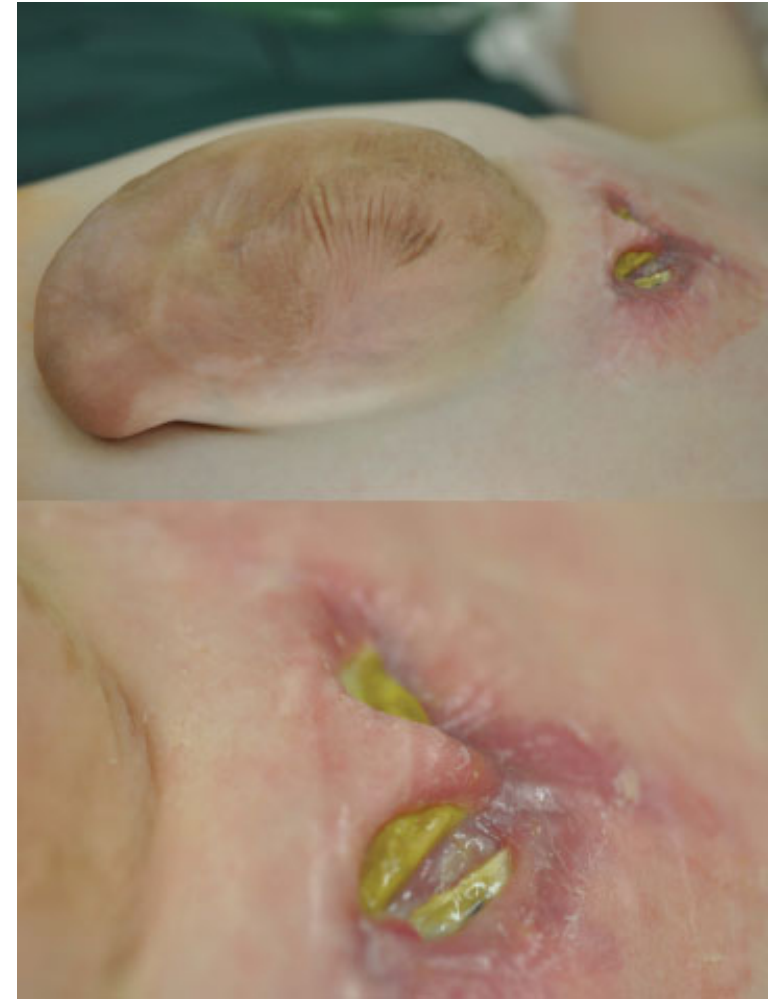

Fig. 1 Eight-month-old patient with omphalocele and wound dehiscence on the anatomic level of normally located xiphoid in the chest area.

The wound dehiscence persisted without any signs for healing. With the child growing and being increasingly disabled by the condition, we planned for a definitive reconstructive procedure at the age of 8 months. To provide a permanent and biological, pliable solution, we opted for a pedicled myocutaneous latissimus dorsi flap for reconstruction of the soft tissue.

\section{Procedure}

The operation started in a supine position. The wound dehiscence over the Gore-Tex patch was removed in the whole exposed area. After removal of the patch, the beating heart was visualized under a thin layer of granulation tissue. Now the patient was repositioned in a right lateral decubital position to mobilize the left latissimus dorsi muscle. Under loop magnification, a pedicled latissimus dorsi flap including a spindle-shaped skin island was elevated. The orientation of the skin island followed a mostly horizontal line to disguise the scar in the later natural bra line. The latissimus dorsi muscle was only on the neurovascular bundle after complete detachment from the humeral insertion (-Fig. 2a-b).

The flap was then transferred through an anterior subcutaneous tunnel after complete release of all muscular attachments from the left axilla toward the anterior defect ( - Fig. 2c-d). After this, an 8-charrier Redon suction drain was placed at the latissimus dorsi donor side, the wound was closed by subcutaneous 4-0 Vicryl (ETHICON, Norstedt, Schleswig-Holstein, Germany) and cuticular running 4-0 Monocryl, and cleaned and dressed with Steri-Strips (3M Deutschland $\mathrm{GmbH}$, Neuss, Nordrhein-Westfalen, Germany). The patient was then again repositioned to the supine position and the flap was adjusted to the defect. Here, the latissimus dorsi muscle was spread over the heart and partly under the local skin and fixed with subcuticular Vicryl 3-0 suture and running 4-0-Monocryl for the cuticular closure. The skin proportion was sufficient and the whole flap well perfused at all times (-Fig. 3). Before wound closure, an 8 -Charriere suction drain was placed below the flap. The patient was ventilated for 36 hours and spent 2 days in the pediatric

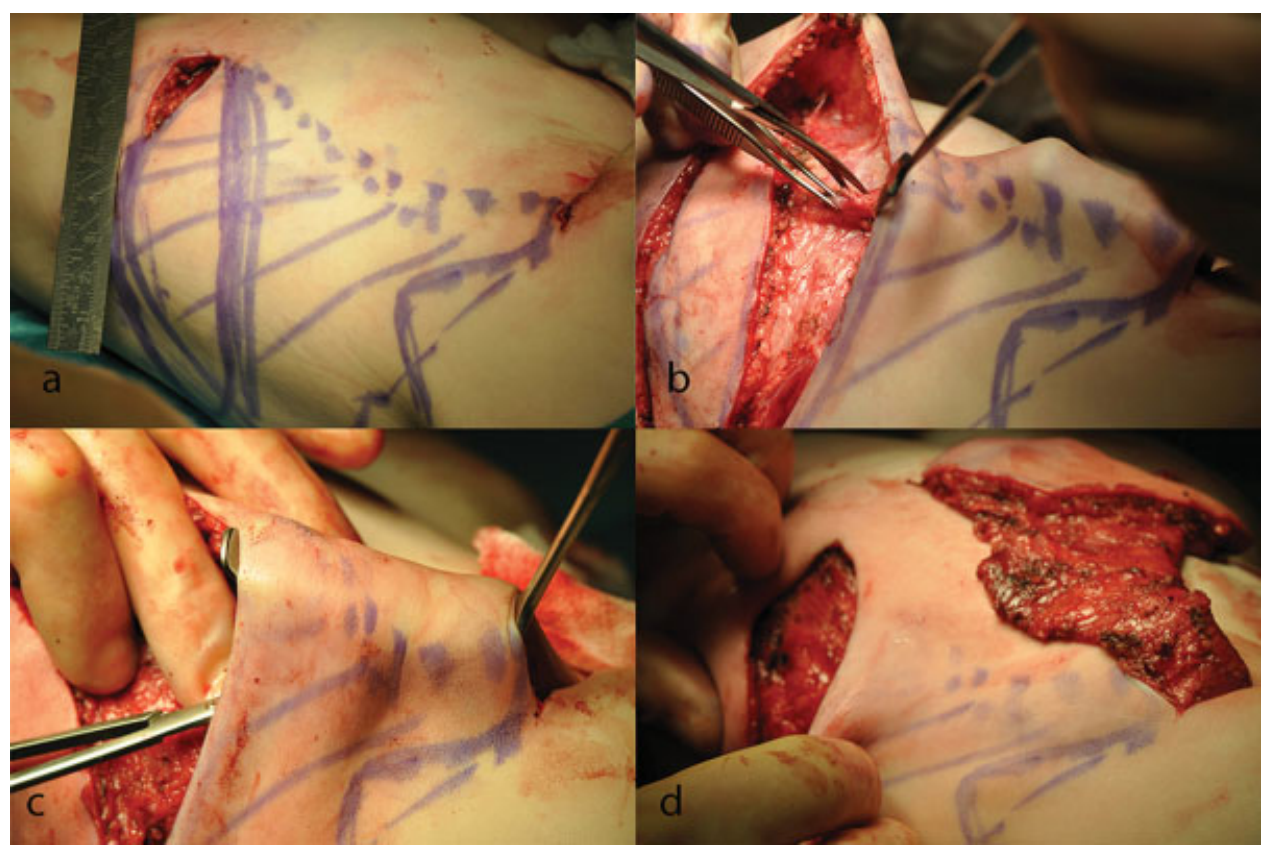

Fig. 2 Intraoperative view. (a) Left part of myocutaneous latissimus dorsi. (b) Patient was repositioned in the right lateral side to mobilize the left latissimus dorsi muscle. (c) Subcutaneous tunnel was created from the left axilla toward the wound location. (d) The flap was rotated through the preformed tunnel to the wound. 


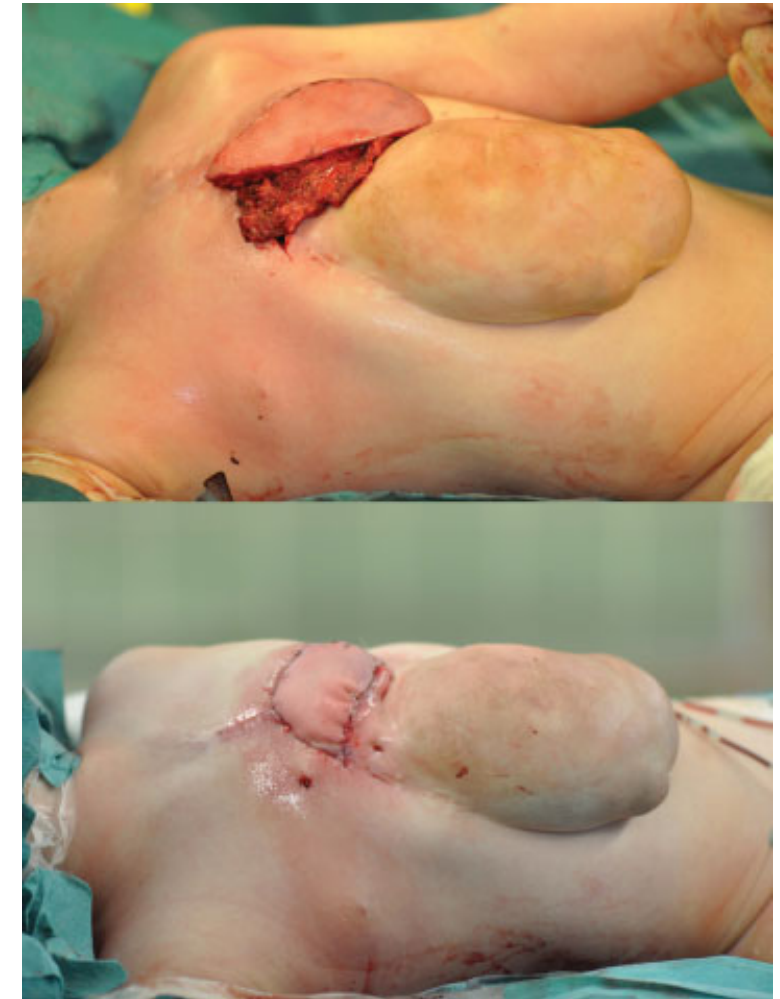

Fig. 3 Intraoperative view. The flap was adjusted to the defect, a drain was placed, and the wound was closed by sub- and intracutaneously suture technique.

intensive care unit. Drains and nonabsorbable sutures were removed at postoperative days 5 and 7. The patient was discharged home on postoperative day 7. The omphalocele was supposed to address at a later age by the pediatric surgeons. In their opinion, EC was the more vital pathology to be treated. After plastic coverage, it became very difficult to have the patient undergo further heart operation because a second operation will not lead to better results. The intracardiac defects remained unrepaired and pulmonary artery banding was used to palliate excessive pulmonary flow; a too tight band arising from physical growth can be dilated with balloon in common interventional radiological procedure.

\section{Discussion}

The cause of EC is still not clarified. Several authors ${ }^{5}, 6$ describe numerous embryonic development failures, which might be reasons for the development of EC: for example, failure of lateral mesoderm in early pregnancy and failure of midline fusion of the developing chest wall caused by compression of the thorax resulting from rupture of the chorion or yolk sac at around 21 days of gestation. ${ }^{1}$ The classification of EC into four types is based on the position of the heart outside the thorax cavity: cervical (3\%), thoracic (60\%), thoracoabdominal (7\%), and abdominal (30\%). ${ }^{2} \mathrm{EC}$ is often associated with Cantrell pentalogy, a syndrome with the following:

1. Defects in the supraumbilical abdominal wall

2. A deficiency in the front of the diaphragm
3. A deficiency in the diaphragmatic portion of the pericardium

4. EC

5. Intracardiac defects described in 1958 by Cantrell et al. ${ }^{3}$

Abdallah et al reported by analyzing series of case reports that $100 \%$ of patients with EC presented with a VSD, $53 \%$ with an ASD, 20\% with tetralogy of Fallot, and 20\% with a left ventricular diverticulum. ${ }^{4}$ These associated defects have a major impact on the treatment and prognosis of EC. However, the general principle for treatment of the ectopic malposition is always the solid covering and protection of the heart, treatment of associated anomalies, and normalization of the body's appearance. The surgical repair of EC seems to be a complex process and it challenges the skills of the surgeons because of its rarity, variations of complexity, and a high death rate. ${ }^{5}$ To offer the best therapy, a multidisciplinary team is needed: a prenatal medical team consisting of a gynecologist, a neonatologist, a pediatric surgeon and cardiologist, a pediatric cardiac surgeon, and plastic surgeons should use their expertise in choosing the best approach to this severe disorder. Particularly, the strategies for chest wall reconstruction are not very uniform and must be modified for each individual case. In previous reports and studies, successful repair of the chest wall defects associated with EC in a newborn has been described using different operation techniques.

Wound closures (primary or otherwise) of sternal clefts have been well described. Vascularized free iliac bone graft with pectoralis muscle flap has been an option for successful treatment of congenital sternal cleft repair. In comparison to an isolated sternal cleft, the repair of the thoracic wall based on EC is associated with greater complexities. ${ }^{7}$

Another option is the use of alloplastic materials. Many authors described various materials used for the reconstruction of the chest wall after replacing the heart into the thorax. The attempt to cover the sternal defect with polytetrafluoroethylene has not been proven successful. ${ }^{8}$ Ley et al reported about covering the thoracic defect after repositioning of the heart by using four methyl methacrylate and Marlex (C.R. Bard, Inc., Murray Hill, New Jersey, United States) composite struts. The final soft tissue coverage was managed with bilateral pectoralis major muscle and skin flaps. ${ }^{9}$ Despite their advantage of easy availability, the unelastic alloplastic materials bear the disadvantages of interference with thorax development, intrathoracic migration, foreign body-related infection, and heart-injury. Alloplastic materials offer valid alternatives for managing the thorax defect by EC, when autologous tissues are not available. However, use of autologous tissues remains the preferable option. ${ }^{9}$

Hochberg et $\mathrm{al}^{1}$ presented a newborn boy with thoracoabdominal EC. After repair of the heart and its reduction into the chest, the wound was covered with bilateral pectoralis major and rectus abdominis myocutaneous flaps combined with intraoperative tissue expansion using Foley catheters. The lateral donor sites were covered temporarily with porcine xenografts. A secondary operation was needed for further corrections. The wound could be closed well; however, the 


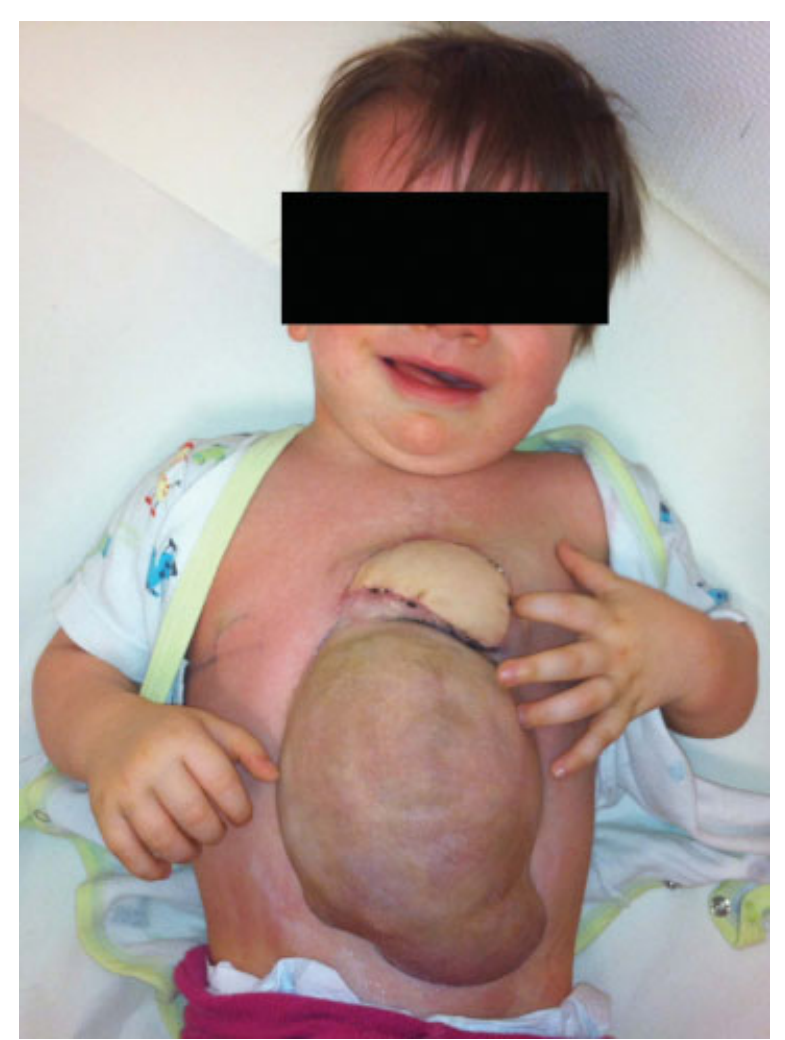

Fig. 4 Postoperative view. Two weeks after the chest wall reconstruction with myocutaneous latissimus dorsi pedicled flap, the child remains asymptomatic.

patient had large scars and the esthetical outcome of this case was therefore inferior. Furthermore, the patient needed a second operation to close the wounds caused by removal of the myocutaneous flaps. ${ }^{1}$ Lampert et al demonstrated successful repair of thoracoabdominal EC using a musculoosseus composite flap involving segments of the 9th and 10th ribs with overlying of pedicled latissimus dorsi muscle. ${ }^{10}$ Bony reconstruction is preferable due to the more stable conditions. However, local infections are at least a relative contraindication. This is why we decided to offer the least invasive and safest approach for a one-step reconstruction and protection of the heart.

In this case, the Gore-Tex patch as a nonvascularized tissue, poor blood supply, tension on the wound margins due to the omphalocele, heart pulsation, and the recurring impairment in pulmonary function maintained infection and wound dehiscence.

The latissimus dorsi flap due to its versatility, constant anatomy, and resistance to infection has become the workhorse in reconstructive plastic surgery. It can be easily harvested in all ages and has a reliable vascular supply by the subscapular vessels.

Therefore, we opted for covering the wound dehiscence with a pedicled latissimus dorsi myocutaneous flap because of its good prognosis for healing and good aesthetical outcome. In addition, it has enough elasticity and stability to support active ventilation, heart beating, and against the distal omphalocele. We recommend the latissimus dorsi flap as an easy and reliable option in cases of complicated reconstruction of EC with previously described problems.

The long-term prognosis of Cantrell syndrome depends on the severity of the cardiac and associated malformations. The treatment of the cardiac malformations, as in our patient, should be performed as required. Treatment of the abdominal malformations should be planned according to their extent and type. An omphalocele that has not ruptured or that is sufficiently epithelialized as in our case does not need an urgent operation but should be repaired early because it is easy to accomplish in a young patient. The next step will be to opt the repair of the associated malformations in consideration to avoid any high abdominal and intrathoracic pressures postoperatively because they cannot be tolerated in the presence of severe cardiac malformations (Fig. 4).

\section{Conclusion}

Autologous tissue for reconstruction of the remaining thoracic defect after repositioning the heart in patients with EC bears the best biological tissue substitute. As in plastic surgical reconstruction of the thoracic wall in adults, the pedicled latissimus flap can also be considered to be a safe and reliable solution for reconstruction of the chest wall in EC after failed mesh repair.

\section{References}

1 Hochberg J, Ardenghy MF, Gustafson RA, Murray GF. Repair of thoracoabdominal ectopia cordis with myocutaneous flaps and intraoperative tissue expansion. Plast Reconstr Surg 1995;95(1): 148-151

2 Sadłecki P, Krekora M, Krasomski G, et al. Prenatally evolving ectopia cordis with successful surgical treatment. Fetal Diagn Ther 2011;30(1):70-72

3 Cantrell JR, Haller JA, Ravitch MM. A syndrome of congenital defects involving the abdominal wall, sternum, diaphragm, pericardium, and heart. Surg Gynecol Obstet 1958;107(5):602-614

4 Abdallah HI, Marks LA, Balsara RK, Davis DA, Russo PA. Staged repair of pentalogy of Cantrell with tetralogy of Fallot. Ann Thorac Surg 1993;56(4):979-980

5 Aliyu L, Mohammad MA. Pentalogy of Cantrell; Complete expression in a nine-month-old boy. Niger Med J 2013;54(3):203-205

6 Gabriel A, Donnelly J, Kuc A, Good D, Doros G, Matusz P, Loukas M. Ectopia cordis: a rare congenital anomaly. Clin Anat 2014 doi:10.1002/ca.22402

7 Engum SA. Embryology, sternal clefts, ectopia cordis, and Cantrell's pentalogy. Semin Pediatr Surg 2008;17(3):154-160

8 Amato JJ, Zelen J, Talwalkar NG. Single-stage repair of thoracic ectopia cordis. Ann Thorac Surg 1995;59(2):518-520

9 Ley EJ, Roth JJ, Kim KA, et al. Successful repair of ectopia cordis using alloplastic materials: 10-year follow-up. Plast Reconstr Surg 2004;114(6):1519-1522

10 Lampert JA, Harmaty M, Thompson EC, Sett S, Koch RM. Chest wall reconstruction in thoracoabdominal ectopia cordis: using the pedicled osteomuscular latissimus dorsi composite flap. Ann Plast Surg 2010;65(5):485-489 